\title{
A Simple Preparation Method for Mesh Fibrin Hydrogel Composites for Conventional SEM
}

\author{
Rebecca E. McMahon ", Mariah S. Hahn ${ }^{*}{ }^{* *}$, Michael W. Pendleton ${ }^{* * *}$ and E. Ann Ellis ${ }^{* * *}$
}

*Department of Chemical Engineering, ${ }^{* *}$ Department of Biomedical Engineering and ${ }^{* * *}$ Microscopy and Imaging Center, BSBW 119/MS 2257, Texas A\&M University, College Station, TX 778432257

Hydrogels have been used extensively in biomedical engineering for scaffolding, drug delivery and many other applications. The high water content of hydrogels makes preparation for electron microscopy challenging. Cryo-scanning electron microscopy (SEM) has been used by some laboratories for examining hydrogels; however, this is an expensive system that only a few laboratories have access to. Some laboratories have used plunge freezing into liquid nitrogen followed by lyophilization and sputter coating. Lyophilization results in shrinkage of the hydrogels. There has been one report of aldehyde fixation followed by dehydration in alcohol and conventional critical point drying in a commercially available critical point drier and sputter coating for hydrogels [1]. This paper presents a simple and cheap alternative method for preparation of hydrogels that can be done in any laboratory. The most critical component is a properly functioning fumehood.

Fibrin hydrogel composites scaffolds were processed by two different protocols. Some specimens were fixed in formalin, plunged into liquid nitrogen and then lyophilized overnight. Cross sections were cut with a razor blade and then the specimen was mounted with the cut surface up on an SEM stub with double sided carbon tape. Stubs were then treated with ruthenium tetroxide vapor for five minutes [2] followed by a light sputter coating with Au:Pd (60:40) and examined by secondary and back scatter imaging in a JEOL 6400 SEM equipped with a tungsten filament at an accelerating voltage of $10 \mathrm{kV}$. Other specimens were fixed with formalin and dehydrated with a graded ethanol series (70-100\%). Final dehydration consisted of three changes of 100\% ethanol with intermittent vacuum (30 sec ON; $30 \mathrm{sec}$ OFF at 15 inches $\mathrm{Hg}$ ) in a PELCO Biowave ${ }^{\circledR}$ microwave system (Ted Pella, Inc., Redding, CA) and a six minute microwave cycle ( 2 min ON; 2 min OFF; 2 min ON) at 200 watts and $25^{\circ} \mathrm{C}$. Ethanol was then replaced with hexamethyldisilizane (HMDS) (Electron Microscopy Sciences, Hatfield, PA) for three changes and the same microwave conditions as done for the final ethanol changes. Cross sections cut with a razor blade were mounted on stubs with double sided carbon tape. Stubs were then exposed to ruthenium tetroxide vapor for five minutes and then lightly sputter coated and examined in the SEM as done for the other samples.

There was significant shrinkage in the lyophilized samples (Figs. 1 and 2) and the samples were soft and spongy when cut with a razor blade. The specimens that were dehydrated with ethanol and critical point dried with HMDS (Figs. 3-6) did not shrink significantly and were firm and crisp when cut with a razor blade. The ruthenium tetroxide vapor treatment yielded well-grounded samples and the use of secondary and backscatter imaging yielded increased information from the specimens.

References

1. M. P. Linnes et al., Biomaterials 28 (2007) 5298.

2. E. A. Ellis and M. W. Pendleton, Microscopy Today 15(3) (2007) 44.

3. This work was supported by grant NSF/CBET 0853668. 

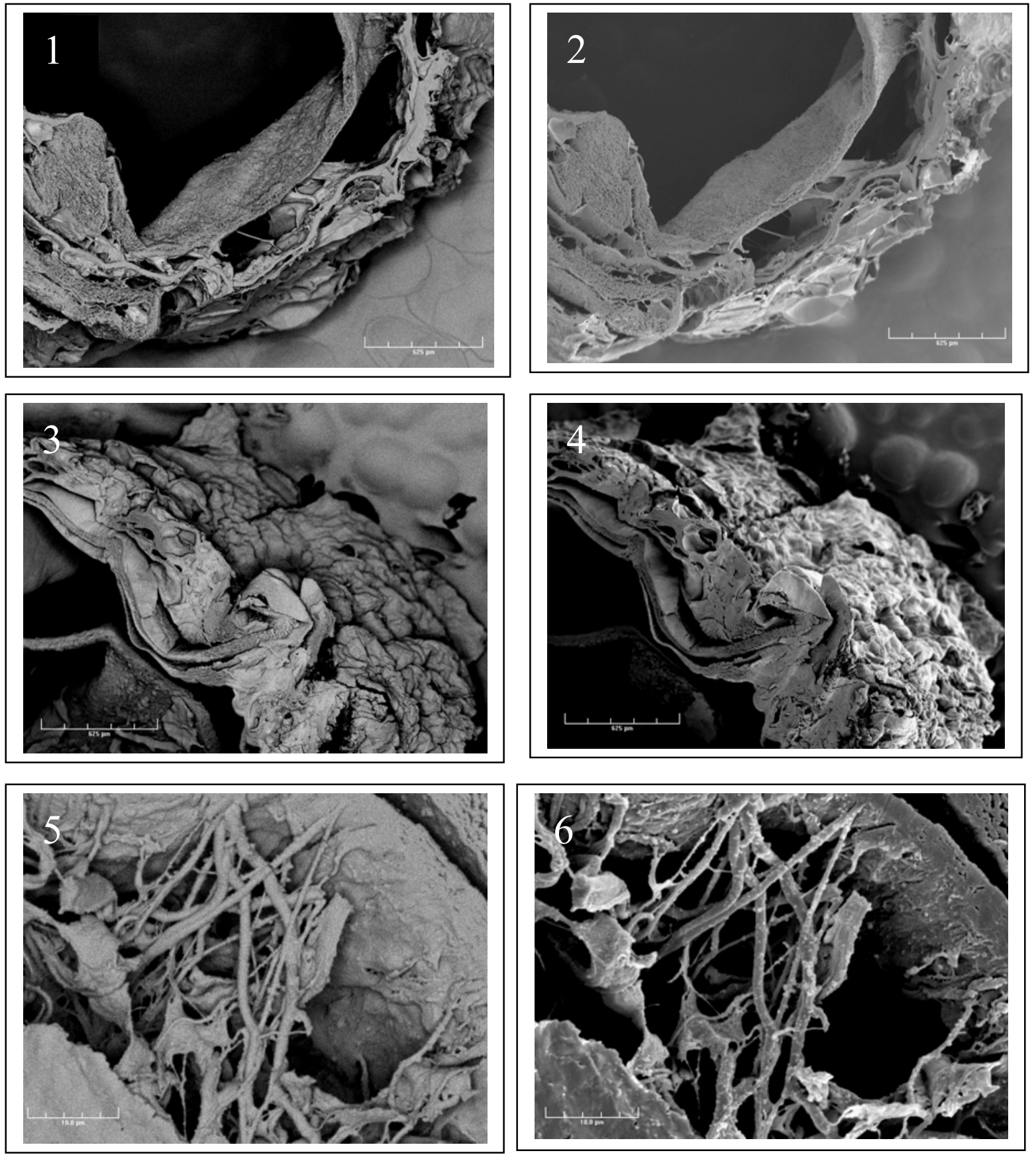

Fig. 1 Backscatter image of lyophilized fibrin hydrogel composite; scale bar equals $625 \mu \mathrm{m}$.

Fig. 2 Secondary image of the same area in Fig. 1; scale bar equals $625 \mu \mathrm{m}$.

Fig. 3 Backscatter image of HMDS treated fibrin hydrogel composite; scale bar equals $625 \mu \mathrm{m}$.

Fig. 4 Secondary image of the same area in Fig. 3; scale bar equals $625 \mu \mathrm{m}$.

Fig. 5 Backscatter image of HMDS treated fibrin gel composite; scale bar equals $30 \mu \mathrm{m}$.

Fig. 6 Secondary image of the same area in Fig. 5; scale bar equals $30 \mu \mathrm{m}$. 